\section{Welche periphere Sättigung ist optimal?}

\author{
Bei der mechanischen Beatmung ist es nicht notwendig, die
} physiologisch maximalen Sättigungswerte anzustreben. Auch ein permissiveres Regime stellt kein Risiko für den Patienten dar, wie die prospektive Multizenterstudie von R. Panwar et al. zeigt. Am J Respir Crit Care Med 2016; 193: 43-51

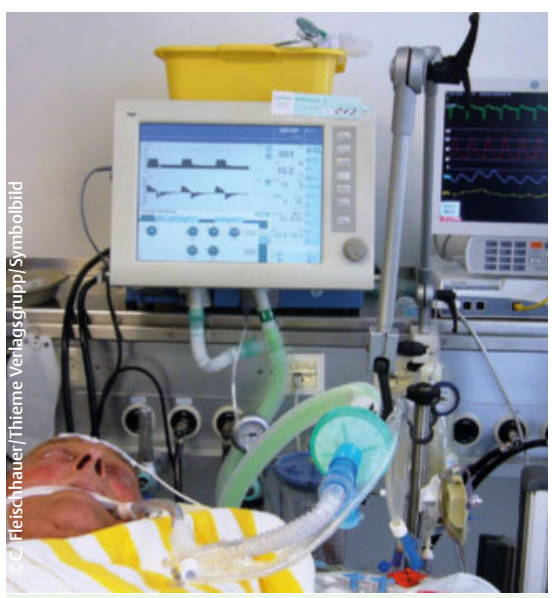

Die mechanische Beatmung ist eine häufig angewandte und lebensrettende Intervention in der Intensivmedizin.

Zahlreiche Aspekte der Intensivmedizin sind empirisch nicht validiert. So war die Frage offen, ob es bei einer maschinellen Beatmung sinnvoll ist, maximale pulsoxymetrische Sättigungswerte anzustreben. Einer an Optimalwerten orientierten Respiratortherapie steht die Möglichkeit gegenüber, auch grenzwertige Sättigungen zu tolerieren. Dann wäre es möglich, toxisch hohe, inspiratorische Sauerstoffkonzentrationen zu vermeiden. Diese Frage wurde bisher empirisch noch nicht angegangen.

Die prospektive Untersuchung erfasste 103 erwachsene Intensivpatienten, bei denen für mindestens 24 Stunden eine maschinelle Beatmung notwendig war. Die Multizenterstudie definierte dabei randomisiert 2 Sättigungsziele:

- Beim permissiven Regime sollte sich der pulsoximetrische Sättigungsbereich zwischen 88 und $92 \%$ bewegen.

- Bei der konservativen Strategie wurde eine Sättigung > 96 \% angestrebt.
Regelmäßige Blutgasanalysen sicherten zusätzlich die Qualität der Beatmung. In der Nachbeobachtungsphase prüften die Autoren, ob die Zuordnung zu einer Sättigungsstrategie den klinischen Verlauf und die Prognose beeinflusst hatte.

Die durchschnittliche, inspiratorische Sauerstoffkonzentration $\left(\mathrm{FI}_{\mathrm{O} 2}\right)$ divergierte entsprechend des gewählten Regimes (konservativ: 0,36, permissiv: 0,26). Die konservativ Beatmeten lagen in 86\% der Zeit im Zielbereich. Unter der permissiven Oxygenierung gelang dies in $97 \%$ der Beatmungszeit. Die Auswertung zeigte, dass sich die Zuordnung zur Sättigungsstrategie weder auf die kurzfristige noch auf die langfristige Mortalitätsprognose auswirkte. Die Kurzzeitmortalität variierte um 37-40\%. Auch die Parameter des klinischen Verlaufs blieben weitgehend unbeeinflusst. Der durchschnittliche Katecholaminbedarf zeigte sich in der permissiven Gruppe leicht vermindert $(0,08$ vs. $0,09 \mu \mathrm{g} / \mathrm{kg} / \mathrm{min}$. Die Aufenthaltsdauer auf der Intensivstation schwankte gruppenunabhängig um 7-9 Tage.

\section{Fazit}

Bei beatmeten Intensivpatienten ist es offenbar nicht notwendig, die physiologisch maximal mögliche, periphere Sauerstoffsättigung anzustreben. Auch die Orientierung an submaximalen Werten im Bereich von 88 bis $92 \%$ bleibt ohne Konsequenz auf die Mortalitätsprognose und den Krankheitsverlauf. Dieser Befund eröffnet die Perspektive, erhöhte, eventuell toxische, inspiratorische Sauerstoffkonzentrationen gezielt zu vermeiden, so die Autoren.

\section{Dr. Horst Gross, Berlin}

Ausschreibung

David-Sackett-Preis 2017

Das Deutsche Netzwerk Evidenzbasierte Medizin e.V. (DNEbM) schreibt den David-Sackett-Preis 2017 aus, der im Rahmen der vom 9. bis 11. März 2017 in Hamburg stattfindenden 18. Jahrestagung des DNEbM verliehen wird. Der Preis zeichnet hervorragende Leistungen auf dem Gebiet der Evidenzbasierten Medizin und Gesundheitsversorgung (EbM, EbHC) in Forschung, Lehre oder bei der Verbreitung der Anliegen der EbM aus.

Die mit $2000 €$ dotierte Auszeichnung wird im Jahr 2017 bereits zum 10. Mal verliehen. David Sackett, nach dem der Preis benannt worden ist, gilt als Pionier der Evidenzbasierten Medizin, die vom angloamerikanischen Raum ausgehend mittlerweile weltweit praktiziert wird. Er gründete 1967 das erste Institut für klinische Epidemiologie in Kanada an der McMaster Universität und später das Oxford Centre for Evidence-Based Medicine. Der im Jahr 2015 verstorbene Sackett war Initiator und langjähriger Mitherausgeber des Journals Evidence-Based Medicine, Autor von zahlreichen Artikeln und Lehrbüchern sowie Begründer der Fortbildungskurse für EbM an der Universität Oxford. David Sackett war Ehrenmitglied im DNEbM.

Mit diesem Preis werden Mitglieder des DNEbM, d.h. Einzelpersonen oder Gruppen von Wissenschaftlern, ausgezeichnet. Interessenten können sich selbst bewerben oder durch den Vorschlag eines Dritten benannt werden. Bewerbungsfrist ist der 31. Oktober 2016. Die Preisträger werden durch eine Jury ausgewählt, die aus Mitgliedern des amtierenden geschäftsführenden Vorstands, ehemaligen Vorsitzenden des Vereins und mindestens einem Patientenvertreter besteht.

Weitere Informationen finden Sie unter www.ebm-netzwerk.de/david-sackettpreis.

Deutsches Netzwerk Evidenzbasierte Medizin e. V., Berlin 